\title{
DAÑO PSÍQUICO Y SU VALORACIÓN MÉDICO LEGAL EN COSTA RICA
}

\author{
Emilson Alfredo Sierra Baca ${ }^{1}$
}

DOI: https://doi.org/10.5377//rd.v42i1.12940

\section{RESUMEN:}

Es relevante poseer conocimiento sobre la valoración médico-legal del daño psíquico, hacer un abordaje de forma general refrescando el concepto de salud mental y sus propias afectaciones. Igualmente conocer agrosomodo: ¿Cómo se clasifica la violencia?, y si ¿Estos diferentes tipos de violencia puede provocar en la psique de una persona un traumatismo o daño psíquico?. Consecuentemente todo daño psíquico tiene sus propios secuelas por ejemplo: las secuelas producidas producto a un entorno violento. De igual modo es trascendental analizar el daño psíquico desde su concepción general y su valoración jurídica, simultáneamente es significativo valorar el concepto de daño moral y sus diferencias con el daño psíquico. Al mismo tiempo es importante conocer la valoración de la Sala Tercera de la Corte sobre lesiones psíquicas de igual modo conocer de forma sucinta el marco legal costarricense con su respectiva valoración médica del daño psíquico. De igual manera es imprescindible apreciar la valoración psicológica del daño psíquico creando conciencia de los efectos producidos por la victimización criminal. Habría que decir el proceso de valoración pericial psicológica. Por última instancia y no menos importante conocer los posibles factores y los efectos psíquicos posteriores a una situación victimizante.

PALABRASCLAVES:Violencia, dañopsíquico, Lesiones Psíquicas, Trauma, Secuelas, psicologíaforense.

Fecha de recepción: $31 / 8 / 21$ Fecha de aprobación: 1/11/2021

1 Abogado, pasante de la Maestría Profesional en Ciencias Penales, Universidad de Costa Rica (UCR).

Correo electrónico: emilson1993@hotmail.es 


\title{
PSYCHIC DAMAGE AND ITS LEGAL MEDICAL ASSESSMENT IN COSTA RICA
}

\author{
Emilson Alfredo Sierra Baca ${ }^{2}$
}

DOI:https://doi.org/10.5377//rd.v42i1.12940

\begin{abstract}
:
It is relevant to have knowledge about the medical-legal assessment of psychic damage, to make an approach in a general way, refreshing the concept of mental health and its own affectations. Likewise, know agrosomodo: How is violence classified? And if these different types of violence can cause trauma or mental damage in the psyche of a person? Consequently, all psychological damage has its own consequences, for example: the consequences produced by a violent environment. Similarly, it is important to analyze psychic damage from its general conception and its legal assessment, simultaneously it is significant to assess the concept of non-pecuniary damage and its differences with psychic damage. At the same time, it is important to know the assessment of the Third Chamber of the Court on mental injuries in the same way to know succinctly the Costa Rican legal framework with its respective medical assessment of psychological damage. In the same way, it is essential to appreciate the psychological assessment of mental damage by creating awareness of the effects produced by criminal victimization. It would be necessary to say the process of psychological expert evaluation. Last but not least, knowing the possible factors and psychic effects after a victimizing situation.
\end{abstract}

KEYWORDS:Violence,Psychic damage,Psychologicalinjuries, Trauma,Aftermath,Forensic psychology.

Date received: 8/31/21

Approval Date: 11/1/2021

2 Lawyer, intern of the Professional Master's Degree in Criminal Sciences, University of Costa Rica (UCR).

Email: emilson1993@hotmail.es 


\section{INTRODUCCIÓN}

La violencia es un fenómeno social, que en el país presenta un aumento, la cifra más actual detalla 341,382 normas de protección de violencia intrafamiliar, la cuales son brindadas para un promedio de 186 medidas de seguridad por día. La suma se da más en mujeres en donde piden resguardo en contra de hombres ya sea (hermano, tío, abuelo, padre, pareja de hogar y demás). Existen diferentes áreas donde se desarrolla la violencia, pero no importa de qué manera, lo importante es que toda persona tiene derecho a vivir una vida sin violencia, y las mujeres no son la distinción. En los diferentes estudios confirman de modo general un vínculo entre la problemática de la violencia y el resultado de dicho acontecimiento, la respuesta de la problemática son las secuelas emocionales y estas se convierten en un daño psíquico en el niño, adolescente, inclusive la vida adulta.

En Costa Rica las diferentes instituciones y programas en donde evalúan el daño psíquico en las víctimas, definen que estas valoraciones son en gran medida importantes para la valoración legal por parte de los tribunales. Donde se penalice y se proceda a una respectiva indemnización, por el daño emocional de la víctima. La evaluación determinada por el psicólogo médico y forense permite determinar en forma específica si existe daño psíquico, lo que procede a evaluar la presencia del daño y las causas que produjeron al resultado. Hacer un análisis de su historia de vida y si se descubren secuelas en la víctima lo que le ha producido una serie de lesiones en su estado emocional, para este se utilizan una serie de herramientas técnicas y prácticas que permitan revelar la demanda.
En el presente artículo se intenta, establecer las valoraciones legales de la administración de justicia, las herramientas y criterios de investigación realizada por los psicólogos forenses necesarias para dar respuesta a una problemática nacional e internacional que atraviesa la sociedad. Por regla general este fenómeno perjudica la salud psicológica cotidiana de la persona víctima de violencia.

\section{METODOLOGÍA}

Para el desarrollo de este trabajo se emplea un método mixto de investigación por una parte tiene un enfoque cualitativo haciendo una breve revisión de la legislación y jurisprudencia costarricense a través de la lectura de documentos fallos, revistas, libros entre otros que sirvan para la investigación.

Conjuntamente haciendo uso del método cuantitativo para ilustrar con datos estadísticos sobre las valoraciones de los estados psicológicos de las personas evaluadas por un especialista.

\section{DAÑO PSÍQUICO Y SU VALORACIÓN MÉDICO LEGAL EN COSTA RICA}

A continuación iniciaremos nuestra temática valorando la significancia de salud mental. Por lo tanto, SALUD MENTAL: Es el bienestar mental, físico, social de una persona, igualmente la podemos definir como la búsqueda constante del equilibrio que nos ayuda a identificar las capacidades intelectuales y destrezas idóneas de cada persona.

En este contexto las personas con incapacidad mental se enfrentan una serie de retos y dificultades relacionadas con el diario vivir. Por esto es necesario referirse al cuidado de la salud 
mental, se debe iniciar a temprana edad, porque es allí donde se desarrollan nuestras principales destrezas, las cuales nos ayudaran en las diversas fases de la vida (infancia, juventud y adultez). La salud mental es un tema que requiere de atención para prevenir todos aquellos efectos negativos que atenten contra el goce y disfrute de una buena salud mental. Porque todos esos efectos negativos conllevan a no poseer una salud mental adecuada, produciendo una serie de dificultades en relación el medio social, afectando el funcionamiento del diario vivir de una persona.

Cuando se daña la salud mental se afecta negativamente todos los proyectos de vida, desarrollándose una serie de limitaciones, por ejemplo no contar con ciertas habilidades y capacidades para el desenvolvimiento cotidiano. Además se debe tener en cuenta si una persona es diagnosticada con el padecimiento de un traumatismo patológico psíquico, se debe asignar una persona que esté pendiente con el cuidado del afectado en los casos de afectación severa. En estos casos principalmente la confianza del individuo es afectada, sumada la falta de exploración con el mundo exterior, teniendo efectos contraproducentes intrínsecos a la estabilidad mental y emocional. Podemos señalar como efectos contraproducentes los siguientes: baja autoestima, pérdida de valores espirituales y morales, entre otros, impidiendo buscar sentido de la vida. Al mismo tiempo hay que tener en cuenta, que el desarrollo emocional se ajusta de acuerdo al entorno donde se encuentra o se desarrolla la persona, juntamente con el estilo de vida que esta posea.

Para definir el término de daño psíquico existe una serie de dificultades. Este estudio se enfoca en el tema de la violencia, factor que produce un daño psíquico y lo cual es un tema de relevancia en nuestra actualidad por la afectación que provoca en la salud mental de las personas. La población se encuentra afectada por este fenómeno, por lo tanto requiere de un debido procedimiento, en donde se efectúe la debida compensación y se penalice por la afectación producida en la víctima, y se efectúe el debido procedimiento en las distintas instancias de justicia. En este sentido es necesario contar con herramientas que efectúen la valoración del daño psíquico, en donde se describan las causas del acontecimiento y los diferentes eventos violentos en donde se produce violencia en la víctima, y como resultado un daño psíquico.

\section{VIOLENCIA Y TRAUMA}

La organización mundial de la salud, define la violencia como el uso intencional de la fuerza o potencia física que origina poder en forma de amenaza hacia una persona, comunidad ogrupo, en el cual se produzcan y medios que pueden provocar muerte, lesiones, daños psíquicos, perturbaciones en el desarrollo o privaciones. (OMS, 2002)

Es decir la violencia son todos aquellos comportamientos y actividades que provocan daños físicos o psicológicos en la vida de una persona, causado un impacto en el individuo afectando el sistema nervioso del mismo. Se puede aseverar los casos violentos afectan de manera negativa originando y desarrollando estrés, donde estas situaciones afectan y ponen en peligro la integridad física y psicológica de la persona. Todo esto conlleva en algunos casos a la formación de traumas derivados de los factores psicológicos y sociales, dependiendo de cierta manera la influencia, la comunicación y el contacto de la víctima con el agresor, en la mayoría de los casos de violencia se encuentran en el núcleo familiar de la víctima. 


\section{CLASIFICACIÓN DE LA VIOLENCIA}

En el estudio de la violencia podemos determinar diferentes tipos de violencia los cuales producen diversos daños psíquicos.

Subraya la utilización de la lista por tipos de violencia, pues define que la violencia física, al ser una irrupción del espacio físico en donde se domina por medio de la fuerza la voluntad de la persona, afecta el espacio emocional. Entonces la interpretación es la violencia física es continuamente violencia psicológica. (Ramírez, 2002)

Es necesario aclarar que toda violencia física posee una conformación con la violencia psicológica, en cambio no toda violencia psicológica mantiene una conformidad con la violencia física. En el caso de la violencia sexual se dan las dos formas anteriores de violencia, se da la incursión en el cuerpo durante la violencia física y el daño psicológico debido al mismo.

a) La violencia física es la agresión en el cuerpo producida por golpes provocando un daño físico, todas estas acciones tienen consecuencias en la víctima, entre ellas la incapacidad para poder recrearse a laborar y en lo social el aislamiento debido a las lesiones externas. Se puede aseverar que también la violencia física produce enfermedades internas derivadas por las lesiones.

b) La violencia sexual es todo acto involuntario que incluye uso de la fuerza en la víctima, para realizar el goce del acto sexual por uno de los intervinientes. También se puede decir que la violencia sexual se puede realizar de dos formas: involuntariamente o inconscientemente, cuando se hace uso de estupefacientes, fármacos, psicotrópicos. Todo esto aprovechando la incapacidad de la persona de proporcionar su consentimiento voluntario.

La violencia sexual se ha asociado con diversos problemas de salud mental, igualmente de conducta en la adolescencia y la edad adulta, en un estudio basado en la población la prevalencia de síntomas o signos que hacen pensar en trastornos psiquiátricos fue de $33 \%$ para las mujeres con antecedentes de abuso sexual $15 \%$ para los que tenían antecedentes de violencia física por parte de su pareja un $6 \%$, para las que no habían sufrido malos tratos, la violencia producto del acto sexual ejecutada por la pareja, aumenta las secuelas de la violencia física sobre la salud mental. (OMS, 2002)

c) La violencia psicológica: Es toda circunstancia que se da cuando se ataca de manera verbal provocando un daño emocional en la persona maltratada. Los daños verbales se originan cuando se quiere desvalorizar o descalificar a una persona provocando una humillación, todo esto conlleva al aislamiento a causa del miedo o intimidación del individuo, deteriorando la salud psicológica de la persona.

Todos estos tipos de eventos violentos provocan un daño psíquico. Además hay que hacer mención, se debe dar un respectivo tratamiento adecuado, aunado la persona pierde la capacidad de manejar el problema, causando un desequilibrio psicológico. 


\section{SOBRE EL "TRAUMA" LO DISRUPTIVO, LA VIVENCIA Y LA EXPERIENCIA}

Detalla que no se puede confirmar con anterioridad que una persona ha sufrido un "trauma" fundamentándose solo en la magnitud, duración y descripción del acontecimiento violento, se debe de valorar la forma en que la persona experimenta el impacto de la violencia y el desarrollo psicológico genera en la víctima, el desenvolvimiento social que este produce y detallar las características definidas basadas en los hechos del acontecimiento, esto quiere decir que un hecho violento no es absolutamente la causa de un trauma, los elementos que hace mención el mismo autor definiendo el trauma van desde: a) lo disruptivo b) la vivencia c) la experiencia. (Benyakar, 2003)

a) Lo disruptivo es la figura fáctica esta permite estudiar y valorar los diferentes tipos de traumas que se dan como ser los accidentes, las violaciones o cualquier situación que provoca un efecto traumático. Por lo tanto se puede aplicar una mejor forma de lo sucedido desde lo fáctico.

b) La vivencia traumática es la forma en que la persona reacciona sobre el evento traumático vivido, y la capacidad que tiene la víctima para procesar dicho acontecimiento.

c) La experiencia es la unión de lo fáctico con la vivencia en este aspecto las personas describen sus experiencias, relatan la forma en que ocurrieron los hechos, y manifiestan la sensación que tienen de los efectos del acontecimiento. Es descripción personal de todos los hechos ocurridos, tiene conexión con la vivencia, pero no interpreta del todo.
Resguarda de la misma manera una extensa escala de sucesos que van más allá del acto físico incluyendo las amenazas e intimidaciones al mismo tiempo la muerte y las lesiones, la definición comprende también las múltiples consecuencias del comportamiento violento, a menudo menos notorio, como los daños psíquicos, privaciones y deficiencias del desarrollo que comprometan el bienestar de los individuos, las familias y las comunidades.

\section{SECUELAS EN LAS PERSONAS VÍCTIMAS DE VIOLENCIA}

Las herramientas internacionales para la documentación de las secuelas de tortura, define los diferentes síntomas como los más frecuentes: re-experimentación del trauma, evasión sentimientos negativos, entorpecimiento emocional, cambios en la autovaloración, depresión, ansiedad, dificultad de controlar impulsos, molestias corporales, perdida de personalidad, disfunciones sexuales (disminución del deseo sexual, disfunción eréctil, disfunción orgásmica vaginismo, y eyaculación precoz, etc.), daño neuropsicológico, abuso de drogas, alteraciones en el sueño y disfunción sexual. (Protocolo de Estambul, 2005)

Las personas en un ambiente de estrés o violencia desarrollan una serie de réplicas corporales que influyen en el funcionamiento del cerebro, en donde se lleva a cabo las diferentes actividades que controlan el funcionamiento corporal del cuerpo humano. En los diferentes estudios de trastorno por estrés postraumático TEPT, se ha centrado en el ordenamiento biológico el sistema límbico el cual incluye en la amígdala cerebral, el hipotálamo su función es disminuir las emociones de la persona. Todo tipo de alteraciones son el resultado de un 
TEPT por ejemplo, si desde la niñez se efectúa un acontecimiento traumático las respuestas son alteraciones en el cerebro humano en diferentes áreas cerebrales, todo esto causa una afectación en la memoria a medida que se desarrolla la persona, y como resultado un trastorno de estrés postraumático. Se ha trazado que el estrés agudo causa el incremento de la concentración y a su vez causa deterioro neuronal en áreas sensitivas del sistema límbico.

En la experiencia traumática, los gráficos mentales que admite entender, proporcionar sentido y procesar un acontecimiento se ven entrecortados, y la persona reacciona con miedo, sin palabras mediante esa imposibilidad de facilitar sentido a la experiencia vivida, angustia a la víctima teniendo como resultado un estado agravado de extrema ansia psicológica. Sin embargo dichos niveles de carga mental dificultan y desordenan las técnicas conocedoras, en el desarrollo del evento fáctico. (Saporta, 1993)

\section{DAÑO PSÍQUICO}

El daño psíquico se puede valorar como todo acontecimiento que perjudica la salud mental de las personas, producto de eventos inesperados $\mathrm{u}$ impactos sorpresivos. Dejando secuelas $\mathrm{y}$ complicaciones en la vida de la persona afectada dentro de su propio criterio individual, laboral y social. Todo esto tiene que ser debidamente evaluado, investigado y resuelto por uno o varios especialistas, realizando una serie de tratamientos relacionados con la psique individual de la persona.

El daño psicológico se define en primer lugar las lesiones psíquicas agudas derivadas por un delito violento en algunos casos, pueden remitir con el paso del tiempo, el apoyo comunitario o un tratamiento psicológico adecuado; y en segundo lugar, a las secuelas emocionales que permanecen en el individuo de forma crónica, como derivación del suceso padecido y que obstaculiza dañosamente en la vida cotidiana. (Echeburúa E., 2004)

Definiendo el concepto de daño psíquico, jurídicamente diremos que el daño psíquico hace referencia a todos aquellos efectos negativos, los cuales producen una lesión mental profunda producida por un resultado brutal. Necesitando la ayuda de un psicólogo, el cual realizara un tratamiento especializado para enmendar los daños encontrados. Adicionalmente se necesita la experiencia y el análisis del perito psicólogo en el área judicial, en donde mediante una evaluación exhaustiva analiza si la víctima tiene indicios traumáticos producto del evento violento, el cual debe ser probado en el juicio. Igualmente se debe considerar una evaluación psicológica, para la valoración de la misma hay que tener una serie de materiales, que ayuden en la examinación del daño psíquico, equivalentemente esto aplica dentro del área judicial. Un tema importante generador de daño psíquico, es la violencia efectuada por diversas formas, causando un evento traumático en el individuo.

\section{DAÑO MORAL}

Es la lesión que sufre un individuo en su honor, reputación, sentimientos o afectos, por acción culpable o dolosa de otros, según el grado de negligencia casualidad o malicia entre el autor y el efecto. (Guerrón, 2017)

\section{DIFERENCIAS ENTRE DAÑO PSÍQUICO Y DAÑO MORAL}

Revista de Derecho. Vol. 42, No. 1, Año 2021|-159 


\section{a) Daño psíquico}

1. Por regla general es considerado de carácter patológico:Elafectadonecesita una valoración médica diagnosticable de cuadro psicopatológico mediante la intervención de un especialista (Perito).

2. Para provocar un daño psíquico no se necesita una situación muy grave. En este sentido cada caso en particular se debe de asimilar de la mejor manera posible.

3. Se necesita una serie de parámetros de evaluación.

4. Su fin es resarcirlo.

5. Es importante tener en cuenta las enfermedades presentes o futuras del afectado para fijar la finalidad.

6. Se debe realizar una minuciosa evaluación con la debida intervención del perito especializado en la materia correspondiente.

7. Buscando realizar la respectiva fijación de la incapacidad.

8. Observar las circunstancias que provocan un trauma psíquico.

\section{b) Daño Moral}

1. En primera instancia su origen no es patológico.

2. No se considera autónomo del menoscabo.

3. Se utiliza en la terminología jurídica sin existir una relación con la psicopatología.
4. Se busca como finalidad una condena.

5. Es importante evaluar la responsabilidad gravosa de la acción realizada por el responsable con el fin de lograr una condena.

6. No se efectúa ningún tipo de prueba, se toman en consideración los hechos idóneos para realizar la respectiva acreditación de los mismos para producirlos.

7. Procede a la situación del reclamante indistintamente del tenor de las lesiones parecidas.

8. La incapacidad no puede no es fijada, ni evaluada.

9. No se requiere de la evaluación de un perito especializado en la materia.

10. Comprende un seguimiento responsable de perjuicio o sufrimiento.

\section{LESIONES PSÍQUICAS}

La lesión psíquica produce cambios clínicos agudos en la persona víctima de acontecimientos violentos, lo que le incapacita para hacer frente a las responsabilidades de la vida habitual tanto en lo familiar, como en lo laboral, social y personal.

Las lesiones psíquicas más usuales son el trastorno de estrés postraumático, cambios drásticos en el estado de ánimo ansioso o depresivo lo que genera un daño en la personalidad de la víctima. 
Por su parte en el análisis sobre lesiones psíquicas en la jurisprudencia nacional (sala tercera de la corte Resolución N. 00283 - 2005) tanto las lesiones físicas y psíquicas se describen como extrema agresividad y afecto, ocasionadas por lo impredecible de los ataques, la repetición de los hechos violentos van originando un deterioro psíquico progresivo lo que destaca los sentimientos de baja estima, la depresión y la sensación de desamparo e impotencia. La falta de motivación obstaculiza afrontar el problema, y tomar iniciativa para enfrentar el problema apatía le impide afrontar el problema y tomar decisiones válidas para salir de ese ambiente.

\section{MARCO LEGAL NACIONAL}

Por su parte en la Acta de Consejo Superior N. 067 - 2016 Protocolo y guía de actuaciones tiene como objetivo establecer los lineamientos para la aplicación del modelo de Justicia reparadora, con atención en violencia doméstica y delitos sexuales, comprendidos en la Ley de Penalización por Violencia hacia las Mujeres y Ley Integral de la Persona Adulta Mayor. Se han adoptado medida para crear una política de género en donde se puedan garantizar los derechos a las víctimas de violencia.

En Costa Rica, a partir de mayo de 1996, la Ley 7586, denominada Ley contra la Violencia Doméstica, entró en vigencia, la cual define de manera concreta la adaptación de cada uno de los tipos de violencia: a) violencia sexual b) violencia psicológica c) violencia física d) violencia patrimonial e) violencia patrimonial. Con relación a la Ley de Protección a Víctimas y Testigos artículo 16 fue comprendida en el Código Procesal Penal, artículos 70 y 71, y constituye una participación, en donde se desempeña el papel para poder compensar indemnizar a la víctima por los daños sufridos protagónica y activa de la víctima, resurgiendo dentro del proceso para ser resarcida de los daños sufridos y de esa manera crear la solución del conflicto.

En cuanto al programa de Justicia Restaurativa es un método de acercamiento y acuerdos con claridad y sencillez. En donde se brinda una respuesta para la solución del conflicto penal, por medio de una estructura adecuadamente organizada en donde se le garantiza a la ciudadanía una resolución segura al conflicto pero principalmente el acceso a una justicia dispuesta y cumplida. Se ofrece la aportación de las víctimas para lograr debida resolución al conflicto, se estima que la reparación del daño sea integral. Las medidas reparadoras deben orientarse a: Sometimiento de tratamiento terapéutico o métodos socio educativos por parte del ofensor que contribuya en el cambio de su conducta mediante los principios de Justicia Restaurativa, en donde integra los valores como herramientas que favorezcan a la paz social mediante métodos colaborativos.

Uno de los delitos más estudiados en Costa Rica es el delito referente a las lesiones o tipos de lesiones tipificadas en el código penal, esta normativa se encuentra regulada en los artículos 123, 124 y 125 tituladas de la siguiente manera: lesiones gravísimas, graves y leves.

En este contexto cuando analizamos el articulado referente a las lesiones del código penal costarricense se observa un acercamiento a la valoración psicológica desde la perspectiva legal. El artículo 123 lo establece de la siguiente manera: "trastorno emocional severo" en donde se impondrá una pena de prisión de 3 a 10 años a quien ocasione que produzca discapacidad intelectual o como lo especifica el artículo 124 
"una debilitación persistente de la salud" en cuanto a las lesiones graves en donde se culpara con una pena de prisión de 1 a 6 años, debemos tener presente la definición establecida por la Organización Mundial de la Salud, cuando se refiere a la afectación de la salud de las personas igualmente el artículo 125 hace alusión a "un daño en el cuerpo o la salud" en donde se especifica como lesiones leves en donde se culpara con una pena de prisión de 3 meses a un 1 año de prisión.

\section{VALORACIÓN MÉDICO LEGAL}

La Valoración Psicológica es un estudio en donde es necesario el conocimiento de las diferentes herramientas psicológicas, en donde se evalúan las afectaciones en la personalidad de la víctima, así como el análisis del comportamiento y la afectación. Este estudio es apreciable en el marco de la justicia en donde se procede a una evaluación forense respectiva al caso, debe realizarse un análisis estricto y competente, y de esa manera determinar las causas y determinar la debida valoración legal.

La incorporación de las pruebas encontradas por el perito constituye la relevancia del caso entre los medios de prueba se encuentran: las fuentes colaterales, las entrevistas psicológicas y los test, la investigación de los expedientes, construyen la base para poder construir el proceso legal, todo esto formula las herramientas probatorias ante el juez, sin embargo los respectivos medios de prueba deben ser reglamentarios. Las respuestas de la investigación psicométrica realizada son compatibles en ciertos casos con el resultado clínico. En la siguiente figura se muestran resultados del estado psicológico de las diferentes fases fisiológicas.

\section{Tabla 1. Prueba SCL-90-R}

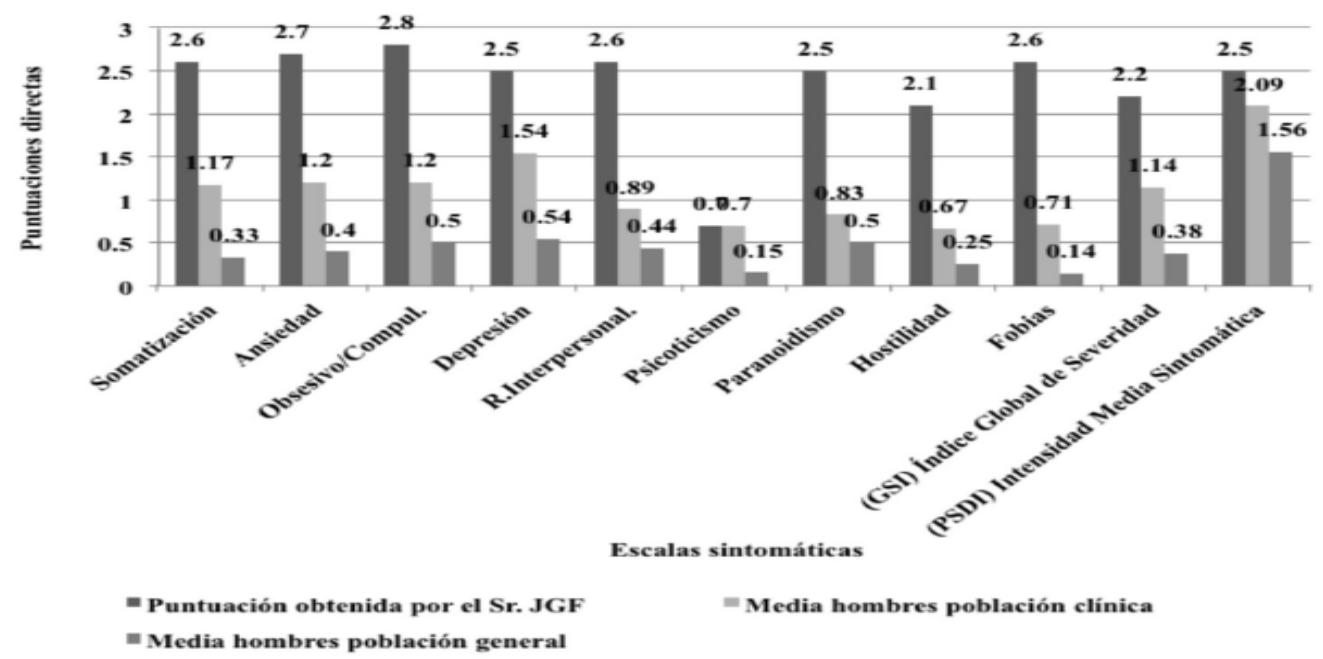

Nota: Resultados obtenidos por el Sr. JGF en la prueba SCL-90-R en comparación con la media de población clínica y población general. (González, 2010) Fuente: SCL-90-R. Cuestionario de 90 síntomas (Derogatis, 2002) 
En cuanto a los test psicométricos los instrumentos que se utilizan en el proceso de evaluación psicológica son los siguientes: El Inventario multifásico de la personalidad MMPI la prueba de psicopatía mide lo que es la desadaptación social, es una prueba muy especificadaencasosmuyseverosdepersonalidad.

La prueba puede llegar a proyectar resultados adecuados con las siguientes características: Depresión, histerismo, hipocon drías,temperamentopsicopático, MasculinidadFeminidad, Esquizofrenia, trastorno obsesivo - compulsivo, Paranoia, problemas para sociabilizarse, autocritica negativa, Capacidad de Liderazgo, porte para laborar bajo presión, interpretación de la realidad, Capacidad para trabajar en equipo con metas en común. (Burgos, 2015) el Test gestáltico visomotor de bender que es un test neuropsicológico, neuropsiquiatría para medir daños cerebrales organicidad de lesiones cerebrales.

Las escalas Wesley se utiliza para medir el coeficiente intelectual tanto en adultos, niños, adolescentes y adultos mayores y que en su gran mayoría se piensa que podían estar en un tipo de demencia, en esos casos se ha aplicado un test de evaluación del estado mental para determinar si la persona está senil o no $\mathrm{y}$, también nos da un promedio de lo que es la inteligencia o cuando la persona manifiesta un tipo de retraso para determinar en qué condición esta para el desarrollo porque también si no es apto para poder desarrollar el peritaje.

Los aspectos importantes son: personalidad, inteligencia, neuropsicología, dificultades de sociabilización. Cada uno de los test está basados en estudios científicos, están estandarizados, están sujetos según su población, según edades, sexo etc.

En resumen los test psicológicos se puede decir que tienen una importancia de cumplir con ciertas directrices, como peritos para poder cumplir los dictámenes, ya que son ese auxilio que tienen los jueces para poder determinar al final su decisión, por la base científica, si bien es cierto todos los casos comparten la misma causal, pero cada caso tiene su peculiaridad ningún caso es igual a otro aunque se comparta conducta patrones o dinámicas, pero cada uno tiene su particularidad y se le da el trato específico a cada uno.

\section{LIMITACIONES}

En la evaluación psicológica se encuentran dos aspectos puestos en crítica, los cuales se relacionan en primer lugar con las fallas técnicas en el diseño de los test, y en segundo lugar los problemas éticos cuando se da la respectiva definición y conclusión de los mismos. Sin embargo lo importante es que los resultados aporten pruebas en el desarrollo de la valoración psicológica en donde la crítica se da en los profesionales que alteran los resultados, y depende de ellos determinar la solución de un caso. (Burgos, 2015)

El Perito en Psicología del área Penal le corresponde figurar en el Informe Psicológico Forense, fundamentos útiles al proceso basándose en lo siguiente: 
Tabla 2. Forma de fijación de la pena

\begin{tabular}{|c|c|c|}
\hline \multirow{2}{*}{$\begin{array}{l}\text { Elementos de } \\
\text { la pena } \\
\text { Elemento } \\
\text { objetivo }\end{array}$} & Contenidos & Pasos para la fijación de la pena \\
\hline & $\begin{array}{l}\text { Se trata de los límites } \\
\text { legales de la pena, } \\
\text { señalados para el } \\
\text { delito cometido. }\end{array}$ & $\begin{array}{l}\text { Primero: Se califica el hecho y se establece la previsión } \\
\text { abstracta de la ley para el delito }\end{array}$ \\
\hline $\begin{array}{l}\text { Elemento } \\
\text { normativo }\end{array}$ & $\begin{array}{l}\text { Lesividad, daño o } \\
\text { peligro, de acuerdo a } \\
\text { la gravedad del hecho. }\end{array}$ & $\begin{array}{l}\text { Segundo: Se establece el límite máximo de la pena para el } \\
\text { caso concreto, atendiendo a la extensión del daño o peligro } \\
\text { para el bien jurídico tutelado, en consideración económica } \\
\text { cuando sea permisible, y vulnerable la víctima, a la } \\
\text { desviación de dolor más o menos básica para la delegación } \\
\text { del hecho y a las circunstancias de modo, tiempo y lugar. }\end{array}$ \\
\hline subjetivo & $\begin{array}{l}\text { Mayor o menor } \\
\text { reproche, en atención } \\
\text { a la mayor o menor } \\
\text { exigibilidad de actuar } \\
\text { conforme a derecho en } \\
\text { el caso concreto }\end{array}$ & $\begin{array}{l}\text { Tercero: ponderación de la culpabilidad en cuanto mayor } \\
\text { o menor exigibilidad de conocer el derecho y de actuar } \\
\text { conforme a ese conocimiento, la oportunidad del delito, } \\
\text { la consideración tenida a la víctima y las condiciones } \\
\text { materiales y espacio-temporales de la comisión del delito. }\end{array}$ \\
\hline
\end{tabular}

Nota: Cuadro Tomado de Resol. N. ${ }^{\circ} 2002-0428$ Tribunal de Casación Penal. San José C.R.

\section{APRECIACIÓN PSICOLÓGICA FORENSE DEL DAÑO PSÍQUICO}

En la actualidad a la psicología forense se le nombra de diversas formas, entre los cuales podemos aseverar: psicología pericial, psicología aplicada a los órganos de justicia, es la derivación de la denominada Psicología Jurídica a la cual se le adjudica el entendimiento y la capacidad para auxiliar a los jueces y tribunales en la determinación de sus actuaciones judiciales.

El psicólogo forense cumple una función muy importante, la elaboración de evaluaciones psicológicas enmarcadas según la operatividad psicológica vinculada con lo jurídico. Por otra parte esta iniciativa de orden técnica tiene sus propios procedimientos evaluativos relacionados con la pericia psíquica, teniendo una relación con el ejecutante jurídico $\mathrm{u}$ apelante por medio del informe psicológico forense, el cual se estima como un medio de prueba en el seno del proceso legal.

La valoración del daño psíquico se adapta intrínsecamente en la jurisdicción penal y es de interés jurídico principalmente por tres aspectos: facilita la calificación del hecho al Ministerio Fiscal, facilita al juez el establecimiento de los respectivos resarcimientos, puede ser tenida en cuenta para fundamentar hechos probados. (Esbec, 2000)

En este contexto es importante mencionar que el perito tiene una función valiosa en realizar sus respectivos análisis psico-legales, porque es el encargado de conocer la normativa jurídica y asociarlo con terminología psicológica. 


\section{PSICOPATOLOGÍA Y VICTIMIZACIÓN CRIMINAL}

En nuestra sociedad es importante evaluar la colisión psíquica producto de la exposición al crimen, teniendo una mayor relevancia para el estudio psicopatológico, por parte del perito, incorporado a la experiencia traumatizante, efectuando sus valoraciones periciales en el caso concreto.

La victimización criminal produce efectos negativos en el afectado, siendo una experiencia traumática, posicionando su vida en peligro conjuntamente con su estabilidad emocional, creando una impresión negativa en el individuo, produciendo falta de estabilidad psíquica. En estos casos es importante mencionar la importancia, que tiene la teoría del estrés transaccional, la cual consiste en una serie de valoraciones mentales relacionadas con el estrés producido en una persona, teniendo como objetivo analizar a los individuos según el entorno que les rodea, relacionado con las valoraciones evaluativas según las exposiciones peligrosas repercutiendo en su estado de confort.

Hoy en día se puede visualizar el volumen del daño psíquico ligado a un hecho de victimización criminal, compuesta por una serie de factores dentro de los cuales podemos mencionar: todos aquellos factores vinculados de una y otra forma con el hecho delictivo, igualmente son asociados los factores de defensa / protección o adaptación, y los factores denominados de vulnerabilidad. De ahí que el tiempo predestinado para realizar las respectivas valoraciones del daño psíquico es el equivalente dentro del marco temporal subsiguiente a la comisión del delito.
Es importante señalar la importancia que juega el cuadro clínico, igualmente es apropiado la participación del psicólogo forense para justipreciar la eficacia de la evidencia del delator, eludiendo circunstancias engorrosas dentro del marco legal. Uno de los ejes importantes a evaluar por parte de los profesionales en la materia es la condición psicológica y psicopatológica referente a los estados de ánimo del examinado, recopilando información para delimitar si existe algún tipo de desorden de la personalidad.

\section{IMPORTANCIA DE IDENTIFICAR UNASIMULACIÓN,SOBRESIMULACIÓN Y DISIMULACIÓN}

Estos tres criterios son importantes en la práctica médica y legal la cual nos ayuda a evidenciar la patología del intervenido, un individuo puede simular un determinado comportamiento para conseguir un favor propio, esto nos lleva a localizar la sobresimulación o imitación de ciertas actuaciones asignadas a un trastorno mental con el objetivo de obtener un beneficio para sí mismo. Por su parte la disimulación es lo opuesto a la sobresimulación, teniendo como meta ocultar de cierta forma cierta sintomatología para alcanzar un aprovechamiento personal. Ejemplo claro de disimulación: una persona que sufra de una cierta patología o trastorno mental debido a la experiencia vivida debido a un estado de victimización oculte de cierta manera su diagnóstico para eludir los procedimientos judiciales, esto se puede efectuar dentro de victimología forense.

\section{PROCESO DE VALORACIÓN PERICIAL PSICOLÓGICA}

Inicialmente se logra identificar una metodología similar entre la evaluación 
psicológica forense y la evaluación psicológica, teniendo como puntos de consenso ciertos atributos vinculados con la actividad técnica. Toda evaluación psicológica se debe delimitar dentro del marco forense, respetando las reglas y principios de otra investigación organizada que admita objetar, exige el planteamiento con su respectivo contrapunto hipotético, comprende un desarrollo para determinar una postura con el objetivo de alcázar una salida temática evaluada.

Por ende es necesario realizar una diferenciación entre las valoraciones forenses con sus respectivas observaciones y el propósito de injerencia terapeuta, aunada los atributos que diferencian el desarrollo del cálculo psicológico especializado. Siendo así que el individuo a examinar, se encuentre en un litigio procesal, es indispensable la valoración de un experto en el área mental y emocional. Donde la persona a evaluar se predispone a contribuir en el proceso psicológico, igualmente se puede considerar como una táctica del abogado defensor para dar a conocer al juez mediante las valoraciones y documentos periciales del evaluado.

De igual manera estar dentro de un juicio produce efectos de ansiedad en los intervinientes del proceso, principalmente aquellas personas que necesitan evaluación psicológica para poder explicar creíblemente el comportamiento de una persona, igualmente evidenciar la alteración mental y emocional del individuo. Igualmente es importante mencionar la repercusión que tiene la elaboración del expediente psicológico forense, el cual puede ser adaptable de una forma negativa en contra del evaluado, debido a la falta de principios morales y éticos logrando de cierta forma un aprovechamiento o eludir un daño. Se debe utilizar un tecnicismo apropiado, para el desempeño psíquico, efectuando una investigación según el propósito pericial conjuntamente con el estudio del investigador en la materia, efectuando dictámenes que ayuden a identificar las respectivas valoraciones adjudicadas a la experiencia experimental.

Un punto muy importante a considerar por parte del especialista (perito psicólogo) referente a la victimología es saber identificar las diferentes tipos de hipótesis, para verificar las descoordinaciones mentales y emocionales de un sujeto examinado. Para determinar su situación hospitalaria, a lo que se le denomina hipótesis, en el supuesto de cuantificación, igualmente existe otro tipo de hipótesis al que se le nombra como hipótesis basadas en el puesto de semejanza la cual consiste en evaluar la situación de la posible enfermedad mental, debido a la exposición de victimización criminal, teniendo cierto paralelismo con la acusación por parte del individuo examinado igualmente el individuo haga acto de presencia en el procedimiento que se le efectuará. Es menester atribuir la presencia del perito psicólogo debido a que su labor se especializa en la construcción de hipótesis, comprobando las mismas con otras efectuadas por otros especialistas de su área. Un punto importante es lo relacionado con él evaluó mental forense es la toma de datos aportados en la entrevista pericial, la cual cumple la función de recopilar información, igualmente la entrevista ayuda a conocer más a fondo las asimetrías del examinado, indicando al perito cuál es el procedimiento a seguir y si debe de cambiar ciertos lineamientos por otros mecanismos clínicos.
XIX. FACTORES PRODUCIDOS POR LOS EFECTOS PSÍQUICOS POSTERIORES A UNA SITUACIÓN VICTIMIZANTE.


Como se ha señalado, el impacto psíquico del delito está modulado por distintos factores que tradicionalmente según Echeburúa se agrupan en: 1 Factores relacionados con la situación de victimización denunciada: parámetros cuantitativos: frecuencia, intensidad y duración, aunados los parámetros cualitativos como ser: la humillación vivenciada por la persona denunciante, lazo de afectividad con el agresor y consecuencias negativas múltiples derivadas del delito 2 Factores de vulnerabilidad: son asociadas a la víctima, respecto al daño psíquico 3 Factores de protección: Son todas aquellas situaciones asociadas a la persona que de alguna $u$ otra forman ayudan atenuar los efectos del delito. (Echeburúa E., Superar un trauma. El tratamiento de las víctimas de sucesos violentos, 2004)

La exposición anterior hace alusión a los factores de vulnerabilidad en los cuales puede recaer una persona después de una victimización criminal, dentro de estos factores de vulnerabilidad podemos mencionar los siguientes:

1. Trastornos mentales, como ser trastorno de la personalidad.

2. Reducido intelecto y comprensión de las cosas.

3. Afectaciones en su autoestima.

4. Se convierte en una persona no abierta al cambio debido al evento traumático.

5. Entre otros.

\section{Dentro de los factores de protección podemos mencionar los siguientes:}

Variables de personalidad: Dentro de estas podemos mencionar todas aquellas actuaciones positivas que tiene un individuo para protegerse de los efectos negativos de una vivencia traumática por la victimización criminal, dentro de las variables de personalidad podemos mencionar: un buen dominio emocional, Tener una mentalidad positiva independientemente de las circunstancias adversas que le ocurran, entre otras.

Igualmente es importante recalcar por regla general toda persona que interpone una denuncia o demanda dentro el organismo judicial, sufre nuevamente una revictimización / victimización secundaria, la cual es producto de asistir a un organismo judicial, recordar que exponer de cierta manera los hechos ocurridos en su contra produce nuevamente un trauma, aunado el sistema judicial vulnera de cierta forma los derechos que le asisten a la persona por ejemplo: la falta de celeridad en el proceso, igualmente la persona afectada no conoce el procedimiento dentro de las instancias judiciales.

\section{CONCLUSIONES}

- La valoración del daño psíquico nos permite conocer nuevas argumentaciones médicas factibles para utilizar dentro de un tribunal, en este sentido es importante crear conciencia de las repercusiones administrativas y legales que repercute en contra de una persona que ha causado una lesión psíquica en un determinado individuo, producto de los diferentes formas de violencia que puede utilizar una persona para traumatizar $\mathrm{y}$ causar daño psíquico en contra de otra.

- En la actualidad hay diferentes manifestaciones de violencia las cuales son utilizadas como formas de agresión en contra otra persona, provocando en la víctima un posible daño psíquico. La víctima deberá ser requerida a una serie de valoraciones psicológicas para diagnosticar los posibles daños psíquicos temporales o permanentes producidos en el afectado. 
- Los entes que imparten justicia deben de brindar el respectivo apoyo a la víctima desde el instante que acude a las instalaciones del tribunal, deben de mantener una adecuada atención en beneficio de la víctima, juntamente contar con el debido personal en las diversas instancias judiciales, evitando una posible revictimización en el afectado.

- La valoración del daño psíquico es muy importante a la hora de impartir justicia ayuda a tipificar el hecho de carácter violento, sancionarlo, reparar a la víctima por los daños, perjuicios y afectaciones causada por la violencia.

- En este contexto la salud mental y emocional es crucial para la atención a las víctimas de violencia y victimización criminal por lo tanto el Estado debe brindar el apoyo financiero, en las instituciones estatales pertinentes adecuado para una mayor atención.

- Es importante que el público tenga conocimiento sobre estos temas de orden educativo, igualmente seguir la pista sobre la valoración del daño psíquico y sus repercusiones desde el punto de vista de la Sala Tercera de la Corte observando un nuevo orden jurisprudencial, ayudando a los estudiantes e investigadores a conocer más sobre la valoración del daño psíquico sin dejar de lado las valoraciones médicas, las cuales ayudan a tener un mejor criterio médico-legal.

\section{BIBLIOGRAFÍA}

- Benyakar, M. (2003). Lo disruptivo. Buenos aires. Biblos.

- Derogatis, L. (2002). Manual. Adaptación por Gonzáles. Madrid. TEA ediciones.
- Burgos, A. (2015). Psicología forense costarricense. San José Costa Rica. Investigaciones Jurídicas S.A.

- Echeburúa, E Amor, P. (2004). Evaluación de daño psicológico en las víctimas de delitos violentos. Psicopatología clínica, legal $y$ forense, 227-244

- Echeburúa, E. (2004). Superar un trauma el tratamiento de las víctimas de sucesos violentos. Madrid. Pirámides.

- Esbec, E. (2000). El psicólogo forense en el proceso penal. Madrid. Edisofer.

- Guerrón, S. (2017). El daño moral. Gaceta jurídica, 1-7.

- Gonzáles, T y Tejero, R. (2010). Valoración pericial psicológica de la victima de mobbing: estudio de un caso. Costarricense de psicología, 35-47.

- OMS (2002). Informe mundial sobre la violencia y la salud. Recuperado de https:// www.uv.mx/psicologia/files/2014/11/ Violencia-y-Salud-Mental-OMS.pdf

- Protocolo de Estambul. (2005). Manual para la investigación y documentación eficaces de la tortura y otros tratos y penal crueles inhumanos o degradantes. Recuperado de https://www.ohchr.org/documents/ publications/training8rev1sp.pdf.

- Ramírez, A. (2002). Violencia masculina en el hogar. El cotidiano, Nro.18, 28-36.

- Saporta, J y Van Der Kolk, B. (1993). Psychobiological Consequences of Severe Trauma en Torture and its Consequences. Great Britain. Cambridge University Press 